\title{
Meeting Abstract \\ MGH Whole Slide Imaging Teleconsultation Practice in Dermatopathology
}

\author{
Nicholas C. Jones, Rosalynn M. Nazarian, Lyn M. Duncan, and David C. Wilbur \\ Department of Pathology, Massachusetts General Hospital and Harvard Medical School, Boston, MA 02114, USA \\ Correspondence should be addressed to Nicholas C. Jones; ncjones@partners.org \\ Received 2 September 2014; Accepted 2 September 2014
}

Copyright (C) 2014 Nicholas C. Jones et al. This is an open access article distributed under the Creative Commons Attribution License, which permits unrestricted use, distribution, and reproduction in any medium, provided the original work is properly cited.

\section{Background}

Nonsubspecialized pathologists frequently request expert consultation in challenging dermatopathology cases. Traditional consultation practice utilizing shipment of glass slides is costly, slow, and of limited educational benefit to the referring physician. Whole slide imaging (WSI) has been suggested as a potential method of overcoming these limitations in the current glass slide consultation practice, but there have been concerns regarding the adequacy of image quality for interpretation of challenging dermatopathology cases. We aimed to investigate the performance of WSI in challenging dermatopathology consult cases.

\section{Method}

52 consecutive clinical consultation dermatopathology cases sent from a community hospital to an academic medical center were sampled and diagnosed by traditional microscopic examination and by whole slide image examination. Matched pairs of diagnoses were evaluated for diagnostic accuracy rates via a masked adjudication process.

\section{Results}

Two of 52 cases (3.8\%) had major discrepancies. After adjudication the WSI diagnosis was preferred in one case and the glass slide diagnosis was preferred in the other. 13 of 52 cases (25\%) had minor discrepancies, with the WSI diagnosis preferred in 6 cases and the glass slide diagnosis preferred in 4 cases and with no preference in 3 cases. Differences in diagnosis were primarily due to interobserver variability and thresholding inherent in challenging dermatopathology consult cases and not due to image quality.

\section{Conclusions}

Overall, the sampled accuracy rates of both WSI and glass slide techniques were equivalent. These results suggest that WSI may be feasible for even challenging dermatopathology consultation cases.

\section{Authors' Contribution}

Nicholas C. Jones and Rosalynn M. Nazarian are cofirst authors. Both authors had equivalent contributions to the paper. 


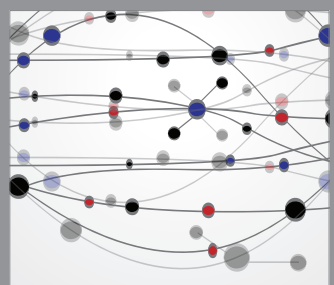

The Scientific World Journal
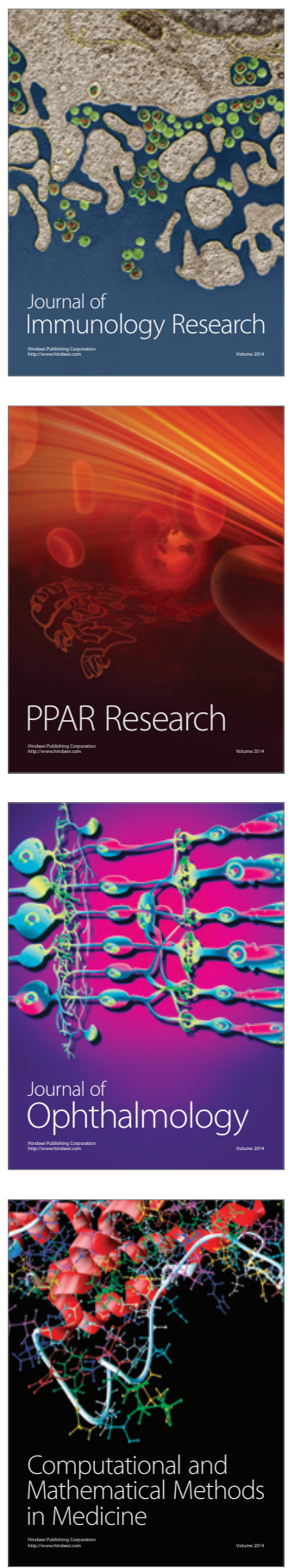

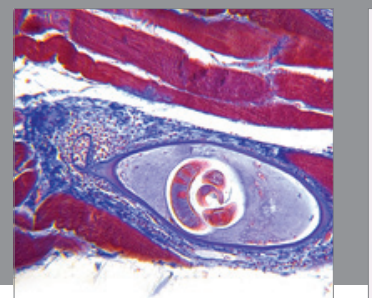

Gastroenterology

Research and Practice
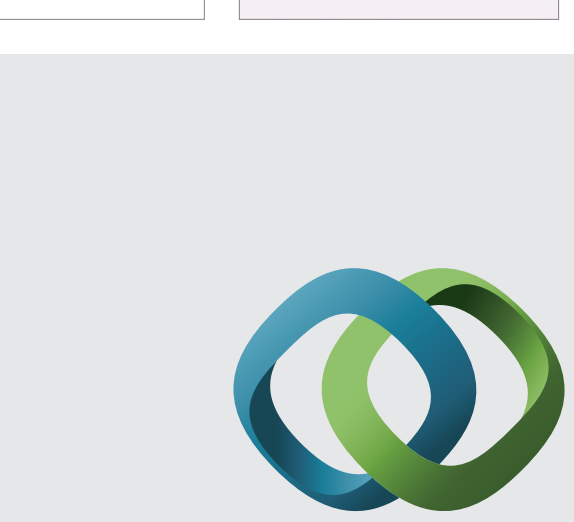

\section{Hindawi}

Submit your manuscripts at

http://www.hindawi.com
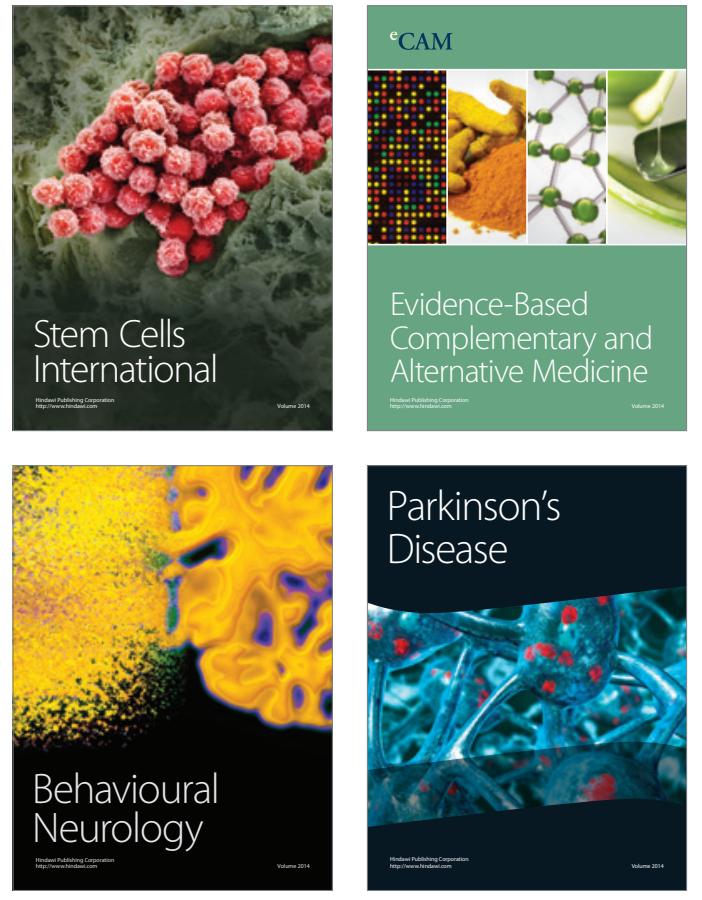
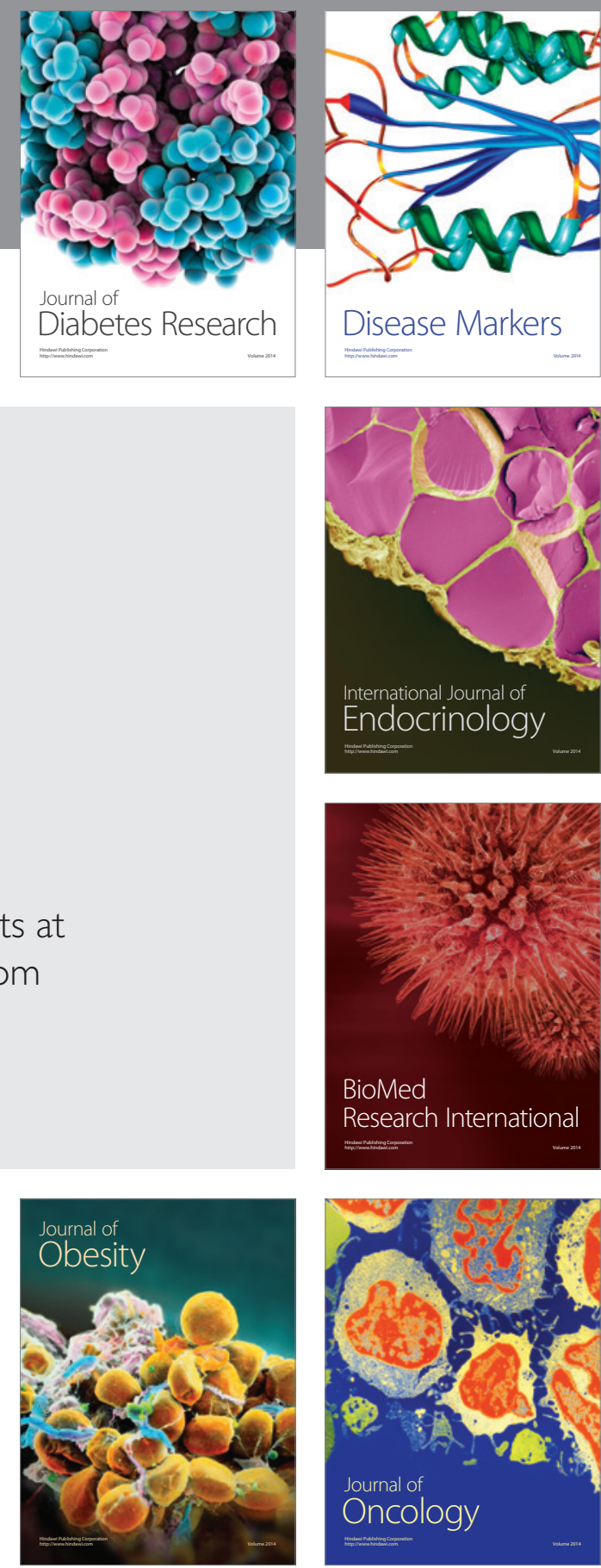

Disease Markers
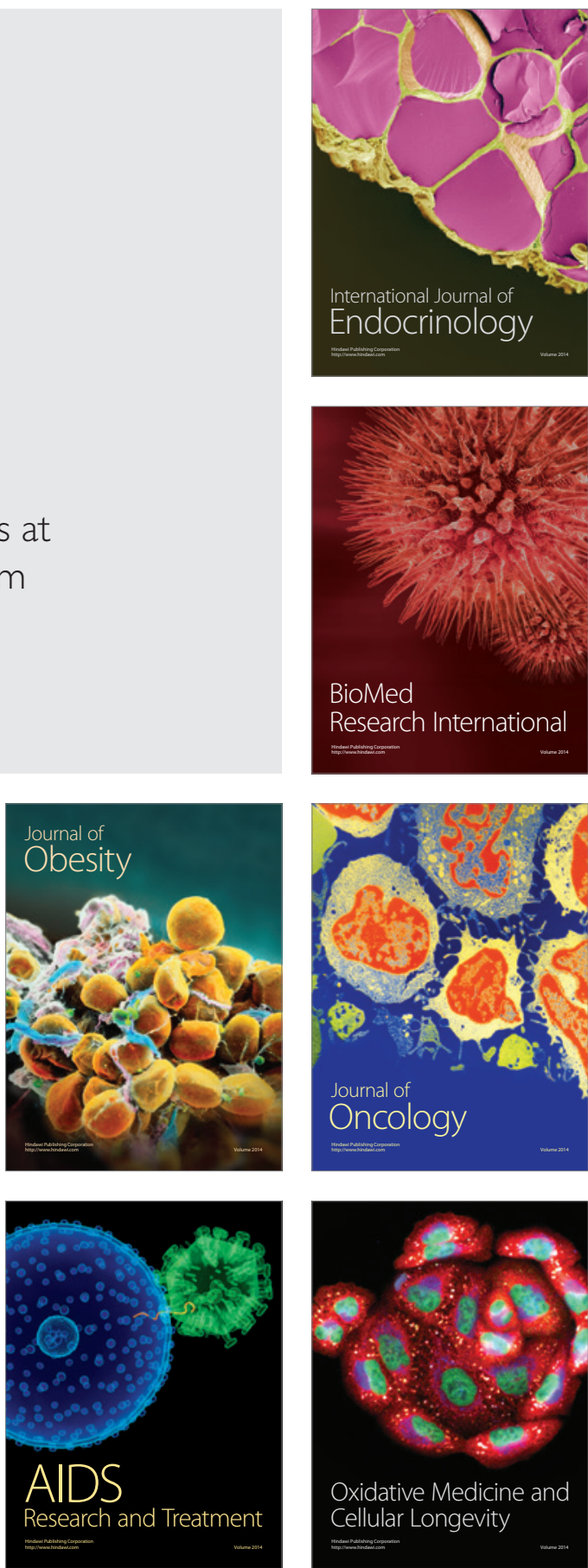